\section{Unexpected benefits and potential therapeutic opportunities of tension free vaginal tape for stress urinary incontinence}

\section{Chendrimada Madhu, Penelope Harber, David Holmes}

Department of Urogynaecology, Gloucester Royal Hospital, UK

\section{Abstract}

Urinary incontinence is a debilitating problem in women with significant effects on quality of life. The tension free vaginal tape (TVT) improves urinary symptoms and achieves a high rate of patient satisfaction. The aim of the study was to evaluate the effect of TVT on vaginal symptoms, pelvic floor function and in turn quality of life. We hypothesize that the TVT is associated with an improvement in pelvic floor function. A prospective questionnaire-based analysis was conducted over a period of ten months in patients undergoing the TVT procedure. The International Consultation on Incontinence Modular Questionnaire - Vaginal Symptoms questionnaire was used to evaluate the vaginal symptoms pre-operatively and 6 months post-operatively. The results were compared to assess for any significant differences. A total of 31 patients were recruited for the study. There was a statistically significant improvement in vaginal symptoms score at 6 months in women undergoing TVT along with an improvement in urinary symptoms. This study suggests a positive impact of TVT insertion on vaginal symptomatology, which is an exciting prospect influencing patient counseling for treatment options of urinary stress incontinence. This finding may have an impact on performing concomitant surgeries for stress incontinence and pelvic organ prolapse. There is a need for further large-scale research to explore this aspect of unexpected benefit from the TVT.

\section{Introduction}

Urinary incontinence (UI) is a common condition affecting 14 to 41 per cent of adult female population. ${ }^{1}$ UI is a distressing and socially disrupting problem that has a significant impact on the patient, her carers and the health service in general. ${ }^{2}$ There is limited information available on the monetary impact of UI in the UK, but data from the US suggests the estimated cost to be over $\$ 12$ billion annu- ally. ${ }^{3}$ UI has a devastating impact on the physical and emotional health of the patient and adversely affects quality of life., ${ }^{4,5}$ Population studies suggest a complex relationship between perceived impact of incontinence and objective measures of its severity. ${ }^{6}$ The current evidence suggests tension free vaginal tape (TVT) as an effective option for managing stress urinary incontinence, with fewer complications and a positive affect on a woman's quality of life. ${ }^{7,8}$ Studies also suggest an improvement in sexual life of women following TVT thus positively affecting quality of life. ${ }^{9}$ There is a paucity of data on the effect of TVT on overall pelvic floor function.

The aim of the study was to evaluate the effects of TVT on pelvic floor functioning, which in turn influences the quality of life. We hypothesize that the TVT is associated with an improvement in pelvic floor function. This prospective study uses the International Consultation on Incontinence Modular Questionnaire - Vaginal Symptoms (ICIQ-VS).

\section{Materials and Methods}

This study was a prospective questionnairebased study of all patients undergoing TVT over a 10-month time period from February 2009 to December 2009. All patients undergoing urogynecological procedures in our unit complete the ICIQ questionnaires, as a part of the monitoring and auditing process. The data is also used as an evaluation of service provision. All the details are stored on the national British Society of Urogynaecologists database. Ethical approval was not deemed necessary, as it was an evaluation of practice. A total of 31 patients were recruited for the study during this period. Informed consent was obtained from all the participants. All the patients were seen in the Urogynecology clinic with urinary incontinence. All the patients underwent urodynamic assessment. Urodynamic stress incontinence with no other abnormality was demonstrated. The patients with detrusor over activity were excluded. The patients listed for TVT procedure were given the ICIQ-VS and ICIQ-UI questionnaires pre-operatively. The same urogynecology team did all the TVT procedures. Questionnaires were sent post-operatively by post and all the women (100\%) returned the questionnaires. Patients in our unit are routinely followed up with postal questionnaires and are not seen in clinic following pelvic operations. The patient demographics, operative details and results from the questionnaires were evaluated further.

\section{International Consultation on \\ Incontinence Modular}

Questionnaire - Vaginal Symptoms

The ICIQ-VS is divided into three sections:
Correspondence: Chendrimada K. Madhu, Department of Urogynaecology, Gloucester Royal Hospital, Great Western Road, Gloucester, Gloucestershire GL1 3NN, UK.

Tel. +44.1159233908

E-mail: chendrimada.madhu@nbt.nhs.uk

Key words: vaginal symptoms, International Consultation on Incontinence Modular Questionnaire - Vaginal Symptoms, tension free vaginal tape, stress incontinence.

Acknowledgements: the authors would like to thank Chris Foy (Medical Statistician) and Dr. Adnan T. Khan for providing help with data statistical analysis.

Conflict of interests: the authors declare no potential conflict of interests.

Received for publication: 28 March 2013.

Revision received: 16 August 2013.

Accepted for publication: 12 September 2013.

This work is licensed under a Creative Commons Attribution NonCommercial 3.0 License (CC BYNC 3.0).

(C) Copyright C. Madhu et al., 2013

Licensee PAGEPress, Italy

Urogynaecologia 2013; 27:e6

doi:10.4081/uij.2013.e6

vaginal symptoms, sexual matters, and overall impact on quality of life. This study analyzed only patient response to the section on vaginal symptoms. The specific questions asked were: 1. Are you aware of dragging pain in your lower abdomen?

2. Are you aware of soreness in your vagina?

3. Do you feel that you have reduced sensation or feeling in or around your vagina?

4. Do you feel that your vagina is too loose or lax?

5. Are you aware of a lump or bulge coming down in your vagina?

6. Do you feel a lump or bulge come out of your vagina, so that you can feel it on the outside or see it on the outside?

7. Do you feel that your vagina is too dry?

8. Do you have to insert a finger into your vagina to help empty your bowels?

9. Do you feel that your vagina is too tight?

The answers are classified into the frequency of occurrence of the symptoms and assigned points accordingly, i.e. never (0 points), occasionally (1 point), sometimes ( 2 points), most of the time ( 3 points), and all of the time (4 points). These points are then used to give a total vaginal symptom score (the higher the total score, the worse the symptoms):

Total vaginal symptom score $=2 \times$ (dragging pain $)+2 x$ (soreness in vagina) $+1 x$ (reduced sensation) $+2 \times$ (vagina too loose) 
$+2 \mathrm{x}$ (lump felt inside) $+2 \mathrm{x}$ (lump seen outside) $+2 \mathrm{x}$ (vagina too dry) $+1 \mathrm{x}$ (fecal evacuation)

Note: Question 9, vagina too tight, is primarily for detecting a potential post-treatment complication and is therefore not included in the official scoring.

\section{Results}

The mean patient age in the study group was 59.6 years (range: 30 to 83 ). A total of 31 patients fulfilled the inclusion criteria during this 10 -month period. The mean VS score preoperatively was 8.96 (SD-7.14). The mean post-operative VS score was 6.45 (SD-5.9). The differences between the two scores were normally distributed. There was a mean improvement of 2.5 in the VS score following TVT at six months post-operatively, which was statistically significant (paired $t$ test; $\mathrm{P}$ value $=0.022536$ ) (Table 1).

\section{Discussion}

This study suggests that tension free vaginal tape not only improves stress urinary incontinence, but also improves vaginal symptoms. Improvements in vaginal symptoms score indicate an improvement in symptoms suggestive of prolapse, which indicates an overall improvement in pelvic floor symptomatology. Since its introduction in 1996 by Ulmsten and Petros, ${ }^{6}$ the TVT has revolutionized the management of urinary stress incontinence worldwide. Several studies have shown that TVT provides a high rate of short-, intermediate- and long-term success. ${ }^{7-12}$ This level of effectiveness has been a measure of change in urinary symptoms, sexual symptoms, patient satisfaction and overall effect on quality of life after the procedure. To our knowledge, based on literature search, there have been no studies looking at the effect of the TVT on vaginal symptoms.

The TVT procedure involves placing a polypropylene tape around the level of the midurethra without elevation, ${ }^{8}$ whereby the main aims are to reinforce functional pubourethral ligaments, thus securing proper fixation of the mid-urethra to the pubic bone and simultaneously reinforcing the suburethral vaginal hammock and its connection to the pubococcygeus muscles. ${ }^{13,14}$ Perineal ultrasound has shown that the tape moves during the Valsalva maneuver from dorsocranial to ventrocaudal position in treated patients. This rotation of the tape against the symphysis, compresses the urethra and the surrounding tissues between the tape and the symphysis, leads to
Table 1. t-Test: paired two samples for means.

\begin{tabular}{lcc} 
& Preoperative & Postoperative \\
Observations & 31 & 31 \\
Mean & 8.97 & 6.45 \\
\hline Variance & 51.1 & 35.06 \\
Standard deviation & 7.15 & 5.92 \\
\hline Degrees of freedom $=30 ; t$ Statistic $=2.405224 ;$ P value $=0.022536$. &
\end{tabular}

augmentation of the pressure on all the structures lying between the tape and the symphysis. Therefore, angulation of the urethra and compression of the urethra between the tape and the symphysis are the main reasons why the TVT corrects urinary stress incontinence. ${ }^{15}$

The mechanisms by which the TVT improves vaginal symptoms may be similar to the way it improves urinary symptoms as described above. The placement of the polypropylene tape unfixed in the suburethral and retropubic spaces helps to support the midurethra ${ }^{16}$ and serves to reinforce the pubourethral ligaments and the suburethral vaginal wall, ${ }^{17}$ which indirectly may increase the functional vaginal space. Pre-operative rotation of the urethra towards the introitus, may give rise to some of the vaginal symptoms. The TVT effectively reduces or prevents this rotation, thereby alleviating those symptoms. There is also the added factor of improvement in urinary incontinence that prevents the local vaginal irritation caused by urine leakage. There is no doubt that the TVT leads to a significant improvement in the general quality of life of these women affected by a debilitating and embarrassing problem. This universal feeling of wellbeing might also lead to a sense of improvement in vaginal symptomatology. Confounding variable like unrecognized prolapse may explain the positive benefits. An experienced practitioner examined all patients in this study and women did not present with any symptoms suggestive of prolapse.

The number of patients in this study is small, but the findings reveal an unexpected benefit of TVT, providing opportunities to explore it further. The result adds another dimension to the understanding and outcome of the TVT procedure, which helps in the process of pre-operative counseling. This finding also has implications when considering concomitant surgeries for stress incontinence and pelvic organ prolapse. It implies that one could delay the surgery for pelvic organ prolapse and proceed with surgery for stress incontinence and evaluate the symptoms postoperatively. Further large-scale studies are needed to evaluate this finding further to make valid claims.

\section{References}

1. Hampel C, Wienhold D, Benken N, et al. Definition of overactive bladder and epidemiology of urinary incontinence. Urology 1997;50: 4-14.

2. National Institute for Health and Clinical Excellence. The management of urinary incontinence in women (internet document/guideline). London: RCOG; 2006. Available from: http://publications.nice. org.uk/urinary-incontinence-cg171

3. Wilson L, Brown JS, Shin GP, et al. Annual direct cost of urinary incontinence. Obstet Gynecol 2001;98:398-406.

4. Hunskaar S, Vinsnes A. The quality of life in women with urinary incontinence as measured by the sickness impact profile. Am Geriatrics Soc 1991;39:378-82.

5. Farage MA, Miller KW, Berardesca E, Maibach HI. Psychosocial and societal burden of incontinence in the aged population: a review. Arch Gynecol Obstet 2008; 277:285-90.

6. Wyman JF, Harkins S, Choi S, et al. Psychosocial impact of urinary incontinence in women. Obstet Gynecol 1987;70: 378-81.

7. Ogah J, Cody JD, Rogerson L. Minimally invasive synthetic suburethral sling operations for stress urinary incontinence in women. Cochrane Database Syst Rev 2009; 7:CD006375.

8. Tincello DG, Botha T, Grier D, et al. The TVT Worldwide Observational Registry for Long-Term Data: safety and efficacy of suburethral sling insertion approaches for stress urinary incontinence in women. $\mathrm{J}$ Urol 2011;186:2310-5.

9. Pace G, Vicentini C. Female sexual function evaluation of the tension-free vaginal tape (TVT) and transobturator suburethral tape (TOT) incontinence surgery: results of a prospective study. J Sex Med 2008;5: 387-93.

10. Nilsson CG, Kuuva N, Falconer C, et al. Long-term results of the tension-free vaginal tape (TVT) procedure for surgical treatment of female stress urinary incontinence. Int Urogynecol J Pelvic Floor Dysfunct 2001;12:S5-8.

11. Doo CK, Hong B, Chung BJ, et al. Five-year 
outcomes of the tension-free vaginal tape procedure for treatment of female stress urinary incontinence. Eur Urol 2006;50: 333-8.

12. Deffieux X, Donnadieu AC, Porcher R, et al. Long-term results of tension-free vaginal tape for female urinary incontinence: follow up over 6 years. Int $\mathrm{J}$ Urol 2007;14:521-6.

13. Ulmsten U, Henriksson L, Johnson P. Varhos G. An ambulatory surgical proce- dure under local anesthesia for treatment of female urinary incontinence. Int Urogynecol J 1996;7:81-6.

14. Petros P, Ulmsten U. An integral theory and its method for the diagnosis and management of female urinary incontinence. Scand J Urol Nephrol 1993;Suppl 153:1-93.

15. Sarlos D, Kuronen M, Schaer GN. How does tension-free vaginal tape correct stress incontinence? Investigation by perineal ultrasound. Int Urogynecol J Pelvic
Floor Dysfunct 2003;14:395-8.

16. Lukacz ES, Luber KM, Nager CW. The effects of the tension-free vaginal tape on proximal urethral position: a prospective, longitudinal evaluation. Int Urogynecol J Pelvic Floor Dysfunct 2003;14:179-84.

17. Meschia M, Pifarotti P, Bernasconi F, et al. Tension-free vaginal tape. Analysis of outcomes and complications in 404 stress incontinent women. Int Urogynecol J Pelvic Floor Dysfunct 2001;12:S24-7. 